\title{
Investigating patterns using cellular automata
}

\author{
László Tóth \\ University of Szeged \\ 13 Dugonics square \\ Szeged, Hungary \\ tothl@jgypk.u-szeged.hu
}

\begin{abstract}
Complex systems based on a simple rule generates random, chaotic patterns. Examples of such models include cellular automata (CA). Over fifty years of CA research have been applied and analyzed in several CA fields, but their network topology study is still not significant.

How a cell can influence its environment is very important in CA models. The chaotic behaviors cause, that the information carried by the system can be of great importance for a pattern that will be passed down through generations.

During our research it was implemented a framework that can determine a communication network formed by Langton's CAs cells. This allows us to analyze factors that can influence the spread of information carried by cells. It is possible to simulate and analyze dynamically changing patterns with the proper parameterization of the developed system. These patterns created by cell cultures are grown in neurobiological labs.
\end{abstract}

\section{Keywords}

cellular automata, information spreading, cellular communication

\section{Acknowledgments}

The author was supported by the EU-funded Hungarian grant EFOP-3.6.2-16-2017-00015. 